J. Neurol. Neurosurg. Psychiat., 1955, 18, 58.

\title{
BASILAR IMPRESSION
}

\author{
BY \\ D. G. PHILLIPS \\ From the South-West Regional Neurosurgical Unit, Frenchay Hospital, Bristol
}

\section{RADIOLOGICAL DIAGNOSIS AND INCIDENCE}

The limits of normality in the level of the uppermost cervical spine relative to the base of the skull are not clearly defined.

Chamberlain (1939) stated that normally the tip of the odontoid is either not elevated above a line from the posterior end of the hard palate to the posterior margin of foramen magnum, or, if it is, not more than a millimetre or so. On the other hand, Saunders (1943) analysed the $x$-ray films taken by a standard technique (with anode film distance of $36 \mathrm{in.)}$ in 100 " normal" subjects, and "the position of the tip of the odontoid ... was found to be $1 \mathrm{~mm}$. below a line from the dorsum of the hard palate to the dorsum of the foramen magnum, with a standard deviation of $3.6 \mathrm{~mm}$.". This meant that in one in five normals the odontoid tip would lie more than $2 \mathrm{~mm}$. above Chamberlain's line, in one in 19 more than $5 \mathrm{~mm}$. above, and in one in 64 more than $7 \mathrm{~mm}$. above the line. Saunders's findings suggest that there is a wide normal variation, and that there is likely to be no precise dividing line between the normal and the abnormal. Any criterion based on Chamberlain's line in the definition of basilar impression would be an arbitrary one.

Bull (1946) raised this objection in suggesting that variation in the inclination of the atlas relative to the hard palate provided a more certain basis for recognizing the pathological case.

Garcin and Oeconomos (1953) mention another radiological criterion described by Fischgold and Metyger. In basilar impression the odontoid and lateral masses of the atlas are elevated above a line joining the tips of the mastoid processes in a normal postero-anterior $x$-ray view of the skull. This line normally passes through the occipito-atlantoid joints.

Cases described in this paper satisfy the criterion that elevation of the tip of the odontoid shall be more than $5 \mathrm{~mm}$. above Chamberlain's line in a lateral radiograph of the skull taken with an anode film distance of 28 in. While it is not argued that this is necessarily the best method, it has the virtue of simplicity. The objection that the posterior margin of the foramen magnum may be difficult to recognize in a plain radiograph can be overcome by tomography. Films were considered adequate for comparison only when taken without rotation of the head, i.e., laterals with the patient "face up" in the supine position and not with the head rotated to one side, a position commonly used for straight radiography of the head.

The figure of $5 \mathrm{~mm}$. has been adopted as aconvenient, though quite arbitrary, dividing line in selecting cases as abnormal. On this basis, during a review of skull radiographs of all patients admitted to the South-West Regional Neurosurgical Unit over a four-year period, eight examples of basilaro impression were found among 612 patients, whose radiographs of the upper cervical spine and skullo. were considered adequate for comparison. This? incidence of approximately one in 76 among neurosurgical patients is much smaller than in Saunders's (1943) series for the same amount of elevation of the odontoid tip in normal cases. While the subject still needs review, it may be concluded that the range of normal variation is not so wide as he suggests.

Two of the cases of basilar impression had been recognized in the period under review. These were particularly gross. In one (Case 18) the whole of the body of the axis with the odontoid and most of the atlas was elevated above Chamberlain's line. In the other, the tip of the odontoid was $15 \mathrm{~mm}$. above the line. In this case the whole of the anterior arch of the atlas was also above the line, its upper margin being $1 \mathrm{~mm}$. higher than the odontoid tip. Greater elevation of the anterior arch of the atlas has been seen in other cases, and is a result of the tilting of the axis stressed by Bull (1946).

Six cases were diagnosed retrospectively as basilar impression as a result of the review.

Elevations of the upper edge of the anterior arch of the atlas and of the odontoid tip above Chamber- 


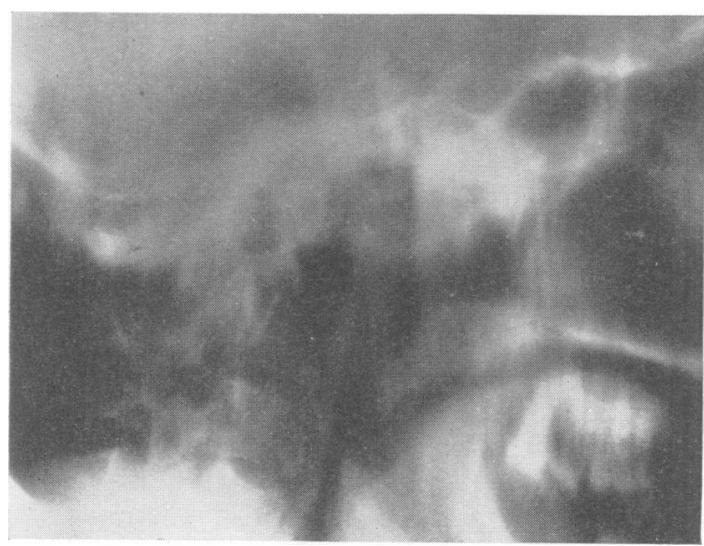

FIG. 1.-Lateral tomogram (Case 19) showing elevation of the odontoid and anterior arch of the atlas.

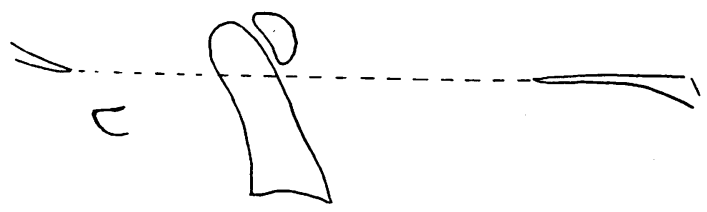

Fig. 2.-Tracing of tomogram in Fig. 1, showing Chamberlain's line.

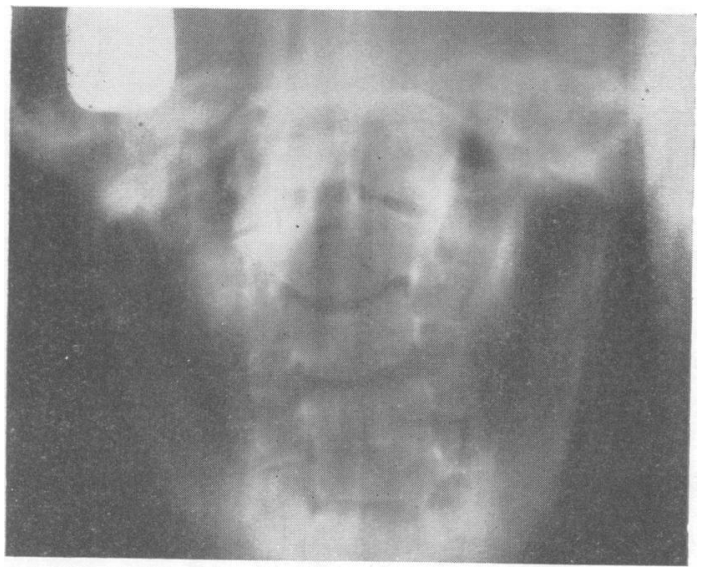

FIG. 3.-Antero-posterior tomogram (Case 19) showing elevation of the occipito-atlantoid joints and the odontoid, also fusion of $\mathrm{C} 4$ and 5 .

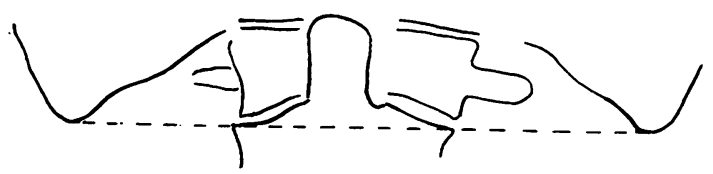

FIG. 4.-Tracing of tomogram in Fig. 3, showing bimastoid line. lain's line in these cases were respectively $15 / 12$, $10 / 10,10 / 10,9 / 10,8 / 8,6 / 6 \mathrm{~mm}$. In another 13 cases diagnosed since, the corresponding measurements have been $14 / 16,10 / 15,15 / 12,13 / 11,11 / 10$, 9/10, 8/9, 6/8, 5/8, 6/7, 6.5/6.5, 6/6, 6/7 mm.

As may be seen, the top of the anterior arch of

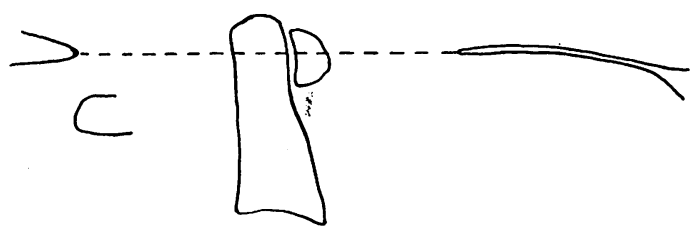

FIG. 5.-Tracing from lateral tomogram (Case 16).

the atlas was elevated to the level of the odontoid tip or above in most of these cases. In some, the whole of the anterior arch was above Chamberlain's line. This is illustrated in Figs. 1 and 2, which show the corresponding angulation of the atlas relative to Chamberlain's line. Antero-posterior views in the same case (Figs. 3 and 4) show elevation of odontoid and lateral masses of the atlas above the bimastoid line.

Fig. 5 is from a case with less elevation of the odontoid and much less tilting of the atlas. Fig. 6 from the same case shows that odontoid and lateral masses are nevertheless well above the bimastoid line.

Even if further researches show that one radiological criterion is more discriminating than another,

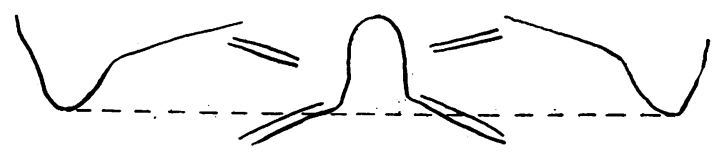

FIG. 6.-Tracing from antero-posterior tomogram (Case 16).

it seems likely that the distinction between normal and abnormal may still remain arbitrary. Meanwhile, the various relationships described above remain of use in a practical way in recognizing cases which may be abnormal and deserve careful study.

\section{ASSOCIATED CONDITIONS}

Basilar impression may be congenital or acquired. The acquired form is most frequently associated with Paget's disease of the skull. One example (Case 20) of this condition was seen in the present series. In another case (Case 18) the basilar impression was secondary to a local bone disease, assumed to be an osteitis, of the atlas and axis. No other case in this series was clearly acquired. A list of associated 


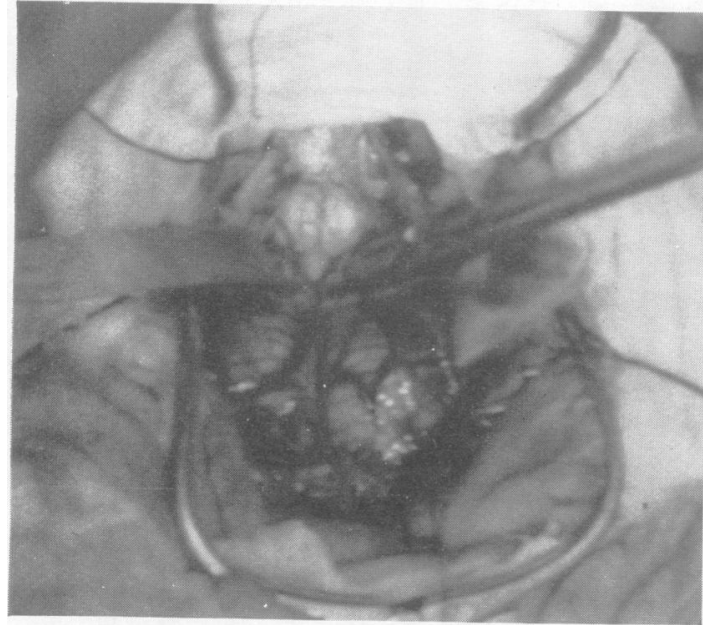

FIG. 7.-Swelling of medulla (Case 1). ? Arnold-Chiari malformation.

lesions known to be present in the cases (Table I) shows, like many other cases reported in the literature, a high incidence of developmental anomalies.

TABLE I

ASSOCIATED CONDITIONS IN 21 CASES OF BASILAR IMPRESSION

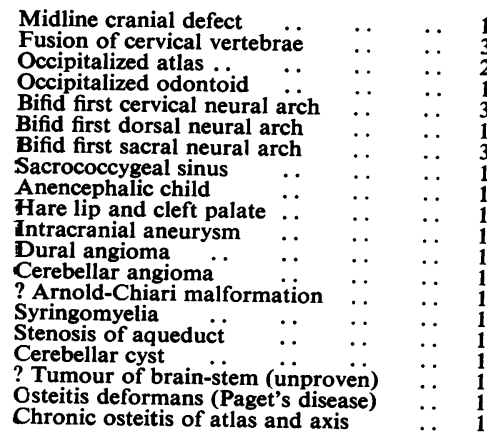

Spina bifida occulta occurs in $17 \%$ of all spine radiographs according to Brain (1951), and in " every tenth man" according to Köhler (1935), but bifid arch of the atlas in unselected cases is less common than spina bifida in general. List (1941) quotes a figure of $3 \%$.

One case of well-marked Klippel-Feil syndrome, or " brevicollis", has been seen in a boy of 7 years with, in addition to irregular fusion and wedging of vertebrae from the third cervical to the fourth thoracic level, a number of other anomalies. These included a midline cranial defect, bifid arches of the sacral vertebrae, fusion of ribs, a sacrococcygeal sinus, and a dropped shoulder (Case 8).
Fusion of two or more cervical vertebrae is discussed at length by Garcin and Oeconomos (1953), who describe it as a " minor form" of the KlippelFeil syndrome. It was present in five of their 18 cases of basilar impression. These authors also found a high incidence of "occipitalization" or "assimilation" of the atlas (seven cases). In one (Case 1) a diagnosis of Arnold-Chiari malformation had been made at operation because of downward displacement of a swollen medulla (Fig. 7) relative to the margin of the foramen magnum, with upward inclination of the uppermost cervical nerve roots. O'Connell and Turner (1950) described this last finding in one of their cases, but did not regard it as proof of Arnold-Chiari malformation. Other authors-Gustafson and Oldberg (1940), List (1941), Lichtenstein (1942), Gardner and Goodall (1950), and Garcin and Oeconomos (1953)-describe this malformation as occurring in association with basilar impression. It is not certain, at least in the case described, whether the soft-tissue deformity was a malformation sui generis or secondary to the bony malformation. This patient had borne an anen=0. cephalic infant, a circumstance which might be्. significant. Recent statistical studies (MacMahon $尺$ 을 Pugh, and Ingalls, 1953) confirm that anencephalu迟 and spina bifida tend to occur in the same fraternity and are likely to be related aetiologically.

One patient (Case 12) had an extensive syringo myelia, proved at necropsy. Lichtenstein (1943) has drawn attention to the presence of true syringo- $\overrightarrow{0}$ myelia as well as other forms of cavitation in the of spinal cord in association with basilar impression.

The diagnosis of congenital aqueduct stenosis was made in one case (Case 3) on ventriculographic evidence, and the symptoms of hydrocephalus had been relieved by a ventriculocisternostomy, the presence of basilar impression being detected only by a subsequent review of the radiographs.

The cerebellar cyst (Case 4) was apparently a benign one, in a patient of 43 years, biopsy of its wall showing only normal cerebellar tissue.

The diagnosis of brain-stem tumour was made in another case (Case 2) on the appearance of a swollen medulla in a ventriculogram and at operation. Unfortunately a post-mortem examination was not available later.

The high incidence of the developmental anomalies (actually distributed amongst 12 cases) argues in favour of a developmental aetiology for basilar impression at least in the cases involved.

\section{CLINICAL FINDINGS}

With this selected material, no statement can be made on the incidence of neurological complications 
of basilar impression in general. It is well known that most cases secondary to Paget's disease have no such complications, though the deformity is often gross. Moreton (1943), in reporting a very large series of 139 cases of basilar impression, stated that there was no absolute relationship between the amount of elevation of the odontoid and the necessity for operation. Study of individual cases in this series confirms this finding.

Six cases, with syringomyelia, stenosis of the aqueduct, cerebellar cyst, presumed brain-stem tumour, cervical spondylosis, and myxoedema, in which the clinical syndrome could be attributed at least in part to the associated lesions, have been excluded from the tabulation of symptoms and signs attributable to basilar impression alone (Tables II and III). So also have three other cases suboccipital pain. In two others there was stiffness of the neck. Discomfort was influenced by jolting (e.g., in a bus) or by change in head posture in five cases. Loss of consciousness followed headaches in three cases.

A flattened, "bun-shaped" head with a short neck was not obvious in most of these cases. Dorsal kyphosis was a prominent feature in some, with increased cervical lordosis.

Cranial nerve disorders were present in less than half the cases. Dysphagia occurred in four, aphonia in two, and trigeminal pain in two.

Cerebellar signs included nystagmus, ataxia, and unsteady gait.

Subjective complaints referred to the trunk and limbs were frequent, but varied in nature and distribution. Where present, pyramidal tract

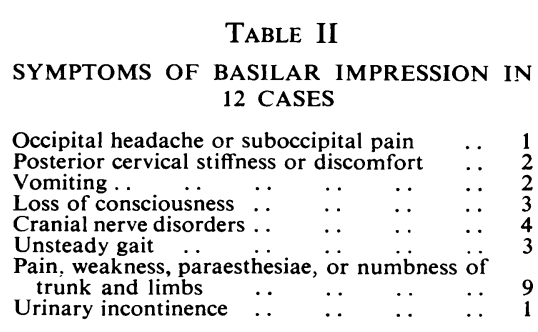

TABLE III

SIGNS OF BASILAR IMPRESSION IN 12 CASES

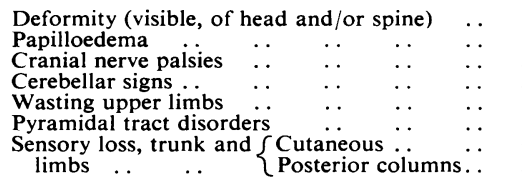

-one of Klippel-Feil syndrome, one of congenital intracranial aneurysm, and another of head injury -in which there were no neurological symptoms or signs attributable to the basilar impression.

Thus there are 12 cases in which the clinical syndrome could be attributed to the effects of the basilar impression alone.

The age of onset of symptoms in these cases varied from 8 to 60 years (average 45 years). Duration of symptoms when first seen varied from two months to 10 years (average two years). In the entire series the sex incidence was 12 male, 9 female.

As pointed out by O'Connell and Turner (1950) and others, symptoms and signs of neurological disorder are due to hydrocephalus, cranial nerve disorders, cerebellar dysfunction, and interference with long tracts.

In seven cases there was occipital headache or 
a congenital anomaly of the atlanto-axial region and excessive mobility there, a partial block was present on flexion only. In six others tested there was no block.

The protein level in the cerebrospinal fluid was elevated to $500 \mathrm{mg}$. per $100 \mathrm{ml}$. in the case with complete block, and to $150 \mathrm{mg}$. per $100 \mathrm{ml}$. in the case with partial block. In others the protein level varied between 15 and $70 \mathrm{mg}$. per $100 \mathrm{ml}$.

Electroencephalography performed in three cases showed diffuse abnormalities. There was little change on repeated testing within three weeks of operation, but in four months post-operatively the E.E.G. was practically normal in one case and improved in another.

\section{CASE HISTORIES}

These have been grouped according to the treatment adopted. It is not implied that finality in choice of treatment has been reached in such cases.

\section{Group 1 : Cases Diagnosed as Basilar Impression by Reviewing Radiographs after Treatment}

Case 1.-G. P., a woman aged 50 years, had had symptoms for eight months. These were suboccipital headaches, vomiting, neck stiffness, papilloedema, and carpal spasms with bouts of suboccipital pain, followed by tingling in the fingers. A suboccipital decompression with laminectomy of C 1-2 was performed. The swollen medulla (Fig. 7) was displaced caudally.

The patient remained well for three years.

A review of the radiographs showed the odontoid tip $10 \mathrm{~mm}$. above Chamberlain's line.

It remains uncertain whether the soft-tissue deformity here was truly an Arnold-Chiari malformation, as diagnosed at the time. Swelling or deformity of the medulla can occur secondarily to a bony malformation. Syringobulbia would be an alternative.

Case 2.-M. M., a woman aged 18 years, had had symptoms for two and a half years. These were headache, diplopia, weakness of the hands and lower limbs, facial paraesthesiae, dysarthria, and dysphagia. Papilloedema, nystagmus, a depressed corneal reflex, facial weakness, palatal palsy, weakness of the tongue, ataxia, increased deep reflexes, and extensor plantar reflexes were found on examination.

A suboccipital craniotomy, with laminectomy of C 1-2 and excision of a venous angioma of the cerebellum, was performed. The medulla was swollen.

After improvement, and relapse five months later, ventriculography showed a persistent swelling of the lower brain-stem. The patient died and there was no necropsy.

A review of the original radiographs showed the odontoid tip $8 \mathrm{~mm}$. above Chamberlain's line.

The absence of post-mortem findings leaves uncertain the nature of the brain-stem lesion, whether tumour, syringobulbia, or other pathology.
Case 3.-B. R., a woman aged 27 years, had had symptoms for six years consisting of headache and tonic fits, with an olfactory aura. She had a large head. Secondary optic atrophy, bilateral ocular palsies, weakness of all limbs, spasticity of the right upper limb, and extensor plantar reflexes were found.

A ventriculocisternostomy was performed after aqueduct stenosis had been demonstrated by ventriculography.

She remained well for three years.

A review of the radiographs showed the odontoid tip $10 \mathrm{~mm}$. above Chamberlain's line.

It is uncertain how much, if any, of the syndrome was attributable to basilar impression. Operative removal of the posterior margin of foramen magnum, and, in this case, the upper two cervical laminae, could have relieved this lesion incidentally in the treatment of the stenosis of the aqueduct.

Case 4.-D. L., a woman aged 43 years, had had symptoms for two years. These were occipital headache, vomiting, paraesthesiae and awkwardness of the left hand, vertigo, and blurred vision. Examination showed kyphosis, short neck, papilloedema, nystagmus, and ataxia.

A suboccipital craniotomy, with drainage of a $\overrightarrow{0}$ left cerebellar cyst, was performed. No tumour was in discovered. A biopsy of the wall of the cyst showed normal brain.

The craniotomy had to be reopened on two occasionso in late convalescence, with laminectomy of C 1-2 an division of the underlying scar, because of persistento loculation of fluid over the posterior surface of the cerebellum.

The patient was well two years later, apart from minos symptoms. A review of the radiographs showed the odontoid tip $6 \mathrm{~mm}$. above Chamberlain's line.

Recognition of the bony anomaly might in the first place have led to a wider decompression and obviated the need for reoperation.

Case 5.-J. R., a woman aged 58 years, had had symptoms for 10 years, namely pain, numbness, and paraesthesiae of the right face. She also had some occipital pain, dysphagia, transient numbness of the right hand, slurred speech, right trigeminal analgesia, absence of corneal reflex (previous alcohol injection for trigeminal neuralgia), and fasciculation of facial muscles.

The trigeminal sensory root was divided and cervical sympathectomy performed.

After initial relief the facial pain recurred. When last heard of, three years later, the patient was a voluntary inmate of a mental hospital.

A review of the radiographs showed the odontoid tip $12 \mathrm{~mm}$. above Chamberlain's line, occipitalization of the atlas, and fusion of the second and third cervical vertebrae. It is suggested that in this case, in which there were confusing psychiatric features, the "atypical trigeminal neuralgia " was only part of a brain-stem disorder. 
Group 2 : Cases with No Symptoms or Signs Attributable to Basilar Impression

Case 6.-A. W., a woman aged 43 years, had had fatal haemorrhage from an intracranial aneurysm.

Later review of the radiographs showed the odontoid tip $10 \mathrm{~mm}$. above Chamberlain's line, and occipitalization of the atlas.

Case 7.-J. W., a man aged 43 years, received a head injury, after which a subdural hydroma was drained. He made a good recovery.

Radiographs showed the odontoid tip $8 \mathrm{~mm}$. above Chamberlain's line.

Case 8.-G. E., a boy aged 7 years, was referred because of a midline cranial defect. The Klippel-Feil syndrome was seen. The odontoid tip was $7 \mathrm{~mm}$. above Chamberlain's line.

\section{Group 3: Cases with Symptoms Subsiding without} Treatment of the Basilar Impression

Case 9.-W. R., a woman aged 44 years, had had symptoms for five months, namely suboccipital pain, vomiting, blurred vision, tinnitus, vertigo, and visual hallucinations. She also had inner ear deafness. Examination showed myxoedema, with an enlarged heart and E.C.G. abnormalities.

Radiographs showed the odontoid tip $10 \mathrm{~mm}$. above Chamberlain's line. The patient improved on thyroid medication, and was symptom-free 18 months later.

Case 10.-W. V., a man aged 47 years, after suboccipital pain, had a sudden unilateral palsy of the tongue, which remained, with atrophy, two months later. There was some limitation of flexion and rotation of the head.

Radiographs showed the odontoid tip $11 \mathrm{~mm}$. above Chamberlain's line, and a bifid first cervical arch.

Case 11.-B. B., a man aged 22 years, had had symptoms for six months, namely headaches, vomiting, diplopia, and unsteady gait. Examination showed nystagmus, diminished corneal sensation, weakness of the left face and right palate, and bilateral spasticity of the limbs.

Radiographs showed the odontoid tip $9 \mathrm{~mm}$. above Chamberlain's line.

Symptoms and most signs subsided after a lumbar puncture in hospital. He remained well six months later.

\section{Group 4 : Cases Treated with Cervical Collars}

Case 12.-A. C., a man aged 40 years, had had symptoms for six months. Two weeks after a fall, pain began at the back of the neck on jolting movements, followed by occipital headache. He had one " blackout". He also had weakness of the right hand and paraesthesiae of the fingers. Pain in the neck on head flexion and rotation, weakness of the hands, and analgesia of the left suboccipital region and ulnar border of the right hand were found on examination.

Radiographs showed the odontoid tip $7 \mathrm{~mm}$. above Chamberlain's line.
Symptoms subsided after the patient had worn a plaster collar for six weeks.

Case 13.-G. A., a man aged 38 years, had had symptoms for four months, namely pain in the shoulders, back, and legs, with paraesthesiae and weakness of the legs. Examination showed short neck, sluggish pupils, weakness of all limbs, wasting of intrinsic muscles of hands, an extensor right plantar reflex, and ataxia. Joint sense in the lower limbs was lost. The chest muscles were weak and there was slight dyspnoea. Unsteady gait had progressed till he was unable to stand unsupported. He had partial spinal block with the head flexed. The C.S.F. contained $150 \mathrm{mg}$. protein per $100 \mathrm{ml}$.

Tomographs showed the odontoid process separate from the body of the axis, and its tip fused with the basiocciput. Chamberlain's line passed through a joint between the base of the odontoid and the body of the axis. This joint allowed the skull and the atlas to move forward on the axis on head flexion.

A Minerva plaster jacket was applied for four months. Immediate improvement resulted, and the patient remained well and was at work 18 months later.

Case 14.-J. C., a man aged 59 years, had had symptoms for four months, namely pain in the left arm, weakness of both hands and lower limbs, and urinary retention. On examination, wasting of the interossei and impaired fine movements of the hands, and extensor plantar reflexes, were found.

Radiographs showed cervical spondylosis. Myelography confirmed narrowing of the spinal canal by osteophytes at the margins of the narrowed disc spaces C 3-4 and 4-5. A radiograph of the skull showed the odontoid tip $6.5 \mathrm{~mm}$. above Chamberlain's line.

There was limited improvement with a plastic collar.

Immobilization was used in these three cases for various reasons. In Case 12 the syndrome was not severe ; it had followed injury (though the relationship was not certain) and the symptoms were aggravated by movement. In Case 13 the need to restrain abnormal mobility of the occipito-cervical junction was clear. Operative exploration with decompression might have further increased mobility and made a bone graft more difficult. In the event, dramatic and lasting relief followed plaster fixation. In Case 14 the collar was applied as treatment for the cervical spondylosis. A Minerva plaster collar was not tolerated. Because of a chronic chest infection, some doubt as to the exact pathogenesis of the neurological lesion, and the patient's age, no operative treatment was considered.

\section{Group 5 : Cases Treated by Surgical Decompression}

Case 15. - R. A., a man aged 20 years, had suffered from frequency and enuresis since childhood. He had to give up work as a hairdresser because of involuntary jerking of the arms. Eleven years before admission he had a total quadriplegia of a few days' duration following 
a fall, then headaches, convulsions, and increasing weakness of all limbs. Nystagmus, wasting of the upper limbs, total analgesia and absence of thermal sensitivity over the upper limbs, upper trunk, and trigeminal area on one side were found. A radiograph of the skull showed a "tam-o'-shanter" deformity, and the odontoid tip was $15 \mathrm{~mm}$. above Chamberlain's line.

A suboccipital decompression with laminectomy of C 1-3 was performed. A mat of adhesions was found obliterating the cisterna magna. Immediately after the end of the operation and removal of the intratracheal tube, respirations became laboured, the pulse slow and weak, the pupils fixed and dilated. The patient's condition improved with coramine, reinsertion of the tube, and oxygen.

He developed spastic flexion of both wrists and metacarpophalangeal joints (cf. Case 1), and then quadriplegia. Bronchopneumonia and meningitis followed and he died two months after operation.

Necropsy showed a true syringomyelia in addition to the basilar impression.

Case 16.-F. P., a man aged 60 years, had had symptoms for eight months. Suddenly he had felt dizzy with a rushing sensation at the back of the head, more on the left. He had several similar episodes, with constant stiffness and discomfort at the back of the neck. Examination showed weakness of the shoulder girdle, unsteady gait, hypalgesia at C2-T9 on the left side, and dorsal kyphosis. Radiographs showed the odontoid tip $8 \mathrm{~mm}$. above Chamberlain's line (Fig. 5).

A suboccipital decompression with laminectomy of C 1-2 was performed.

After three weeks of good progress with improvement in symptoms and signs the patient developed resistant meningitis and died two months later of bronchopneumonia.

Necropsy showed, in addition to indentation of the lateral lobes of the cerebellum by raised lateral margins of the foramen magnum, that the lumen of the right vertebral artery was obliterated at foramen magnum level by arteriosclerosis.

Case 17.-D. K., a woman aged 41 years, had had symptoms for one year. These were attacks of suboccipital headache followed by loss of consciousness (no convulsions) and weakness of the left limbs. Examination showed slight left spastic hemiparesis and hemianalgesia. Radiographs showed the odontoid tip $10 \mathrm{~mm}$. above Chamberlain's line. Ventriculography was normal.

A suboccipital craniotomy with laminectomy of C 1-2 was performed.

Symptoms and neurological signs disappeared immediately. She was well, apart from minor symptoms, over a year later.

The progress of the next two cases under treatment is described in more detail, as they illustrate clearly their peculiar problems. Case 18, at first undiagnosed, developed an acquired basilar impression to a remarkable degree, with very severe neurological signs. Treatment was prolonged, but apart from the immediate post-operative period, recovery was uninterrupted. Case 19 did not present such a formidable picture, neurologically or radiologically, but the post-operative course was stormy.

Case 18. - A man aged 53 years was first seen in November, 1949, with meningismus and some C.S.F. pleocytosis (cells 193 per c.mm., 70\% polymorphs). There were subsequently some dysphagia, hoarseness, and slurring of speech, and poor movement of the palate and pharynx and pharyngeal anaesthesia. Biopsy of an enlarged cervical gland showed " reactive inflammatory hyperplasia".

He recovered from this acute illness and returned to work, but was seen again in February, 1951. Increasing weakness of the limbs since October, 1950, had progressed rapidly almost to complete quadriplegia. There was gross wasting of the shoulder girdle and trapezii

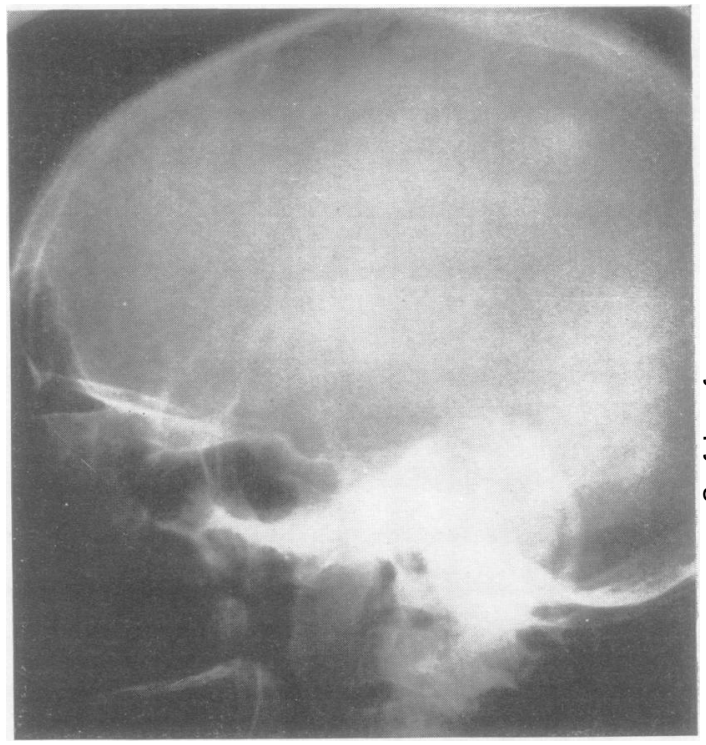

Fig. 9.-Extreme basilar impression : all of body of $\mathrm{C2}$ above Chamberlain's line; retropharyngeal soft-tissue swelling (Case 18).

with severe weakness in these muscles and the sternomastoids. He had faecal and urinary incontinence. The voice was very weak. Radiographs showed gross basilar impression, i.e., the odontoid and what could be seen of most of atlas and axis were pushed upward and indeed were obscured in the lateral $x$-ray view by the mastoid air-cells (Fig. 9).

A review of the radiographs of November, 1949, showed that there was some degree of basilar impression at that time, the odontoid tip being $10 \mathrm{~mm}$. above Chamberlain's line, though it had increased in degree since. Now it also appeared there was some disorganization of the odontoid and soft-tissue swelling in front of it in the post-pharyngeal region. Biopsy of this swelling showed chronic granulation tissue. 
Some immediate improvement followed traction with skull callipers, and five weeks later suboccipital decompression was performed with laminectomy of C 1-2 under local anaesthesia. The medulla and uppermost cord were seen to be dorsally displaced. Skull traction was maintained during the operation and while the patient was removed from the table to a plaster shell, care being taken to avoid twisting the head.

At this stage the patient abruptly lost consciousness and respirations became very slow and periodic. Respirations improved after a dose of methedrine intravenously, and on being given a second dose, about 20 minutes after being moved from the operating table, the patient suddenly regained consciousness and respirations became normal.

Further recovery was slow but steady. The patient remained another 11 months in hospital, during which time he had a more extensive laminectomy of C 3-5 and a rib graft to the occiput and lower cervical spine. $\mathrm{He}$ has since made a complete neurological recovery and was well (apart from a stiff neck) and at work in July, 1954 (Fig. 10).

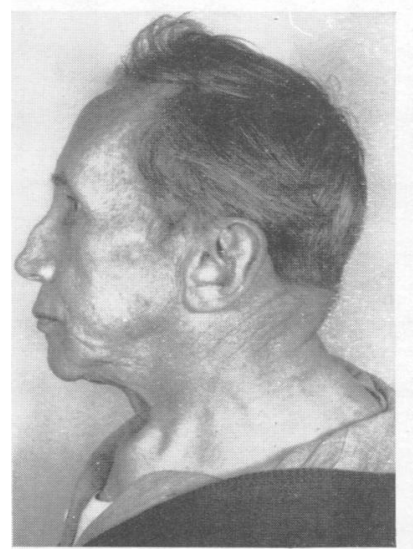

Fig. 10.-Extreme basilar impression : shortening of neck (Case 18).

The origin of the osteitis which may have supervened and aggravated a pre-existing basilar impression is obscure. The nasal septum was largely destroyed, and the patient had a history of treatment for syphilis, though details were lacking and the Wassermann reaction was now negative in blood and cerebrospinal fluid.

Case 19. - A man aged 37 years had had symptoms for five years, consisting of pain in the neck and left upper limb, tremor of the head and upper limbs, and giddy attacks. The odontoid tip was elevated $12 \mathrm{~mm}$. above Chamberlain's line. There were asymmetry of the skull, distortion of the foramen magnum, and fusion of the fourth and fifth cervical vertebrae (Figs. 1-4).

Recent episodes of severe headache followed by unconsciousness led, after careful consideration, to a decision to operate, despite the fact that after a period of rest in hospital the patient was symptom-free and had no abnormal neurological signs apart from slight tremor and slowness of gait.

Suboccipital decompression with removal of the arch of the atlas was performed under general anaesthesia. Inversion of the margins of the foramen magnum was marked. The cerebellar tonsils were small, flattened, a little prolapsed, and very closely adherent to the lower medulla. The junction of the medulla and uppermost cervical cord was angled dorsally and rotated a little. The foramen of Magendie was obstructed by a transparent membrane through which was made an opening into the fourth ventricle.

The patient failed to recover consciousness after being moved to bed and stopping the anaesthetic. There was some fasciculation of the face on one side. The respirations were periodic with long periods of apnoea. This improved somewhat with intravenous and intramuscular coramine and oxygen, but no tone returned to the jaw muscles. Laryngoscopy was very difficult. The aditus laryngis was apposed to the posterior pharyngeal wall, which was unduly prominent because of a marked cervical lordosis. There was seen to be some unilateral fasciculation of the tongue. Finally, as the airway had been maintained only by strongly holding the jaw forward, an intratracheal tube was reinserted and oxygen administered.

Five hours after the end of the operation the patient was moving the right upper limb, and respirations were straining in character. A tracheotomy was performed. The patient remained semi-stuporose for the next two days and developed a severe chest infection requiring frequent aspiration of profuse secretion from the air passages.

Three weeks after the cranial operation, gastrostomy was performed because of difficulty with an indwelling oesophageal feeding tube, which caused local irritation in the nose and throat and did not prevent inhalation of secretions and regurgitated feeds. Daily lumbar puncture drainage had to be performed because of persistent accumulations of fluid in the suboccipital region. Over the next month his condition slowly improved and the tracheotomy and gastrostomy tubes were removed.

Seven months after operation there was still marked unsteadiness of gait, though tremor had virtually disappeared and the plantar reflexes, previously extensor, had become flexor.

\section{Group 6 : Cases Left Untreated}

Case 20.-C. P., a woman aged 58 years, had had symptoms for 18 months, namely tinnitus, pains in the head and right face, dysphagia, and "giddy turns". On examination a large head, deafness, exaggerated tendon jerks, an extensor left plantar reflex, hypalgesia of the right upper limb, and impaired joint sense were found.

Radiographs showed advanced Paget's disease of the skull, and the odontoid tip $16 \mathrm{~mm}$. above Chamberlain's line.

This patient was not entirely incapacitated, despite a variety of symptoms. It was not clear that all these could be attributed to deformity of the foramen magnum. 
Finally, her cardiovascular state contraindicated any but the most urgent surgery.

Case 21.-A. G., a man aged 56 years, had had symptoms for five years, namely, attacks of tonic spasm of trunk and limbs, weakness of the legs, and dysphagia. $\mathrm{He}$ had been bedridden for over a year. Examination showed limited ocular deviation, dysarthria, wasting of the shoulder girdle, and a spastic right hemiparesis. He was incontinent. He had a hare lip and cleft palate. The testicles were not descended.

Radiographs showed the odontoid tip $6 \mathrm{~mm}$. above Chamberlain's line.

Here there was an advanced neurological syndrome with a relatively slight basilar impression. This suggested the possibility of an associated soft-tissue pathology, rather than a simple compression due to a bony anomaly. His physical state was very poor, and it was considered that any surgery would be attended by grave risk without likelihood of benefit.

\section{TREATMENT}

The results of treatment are shown in Table IV. It is clear from a study of the cases described that no routine prescription can be laid down for their handling.

TABLE IV
RESULTS OF TREATMENT IN 21 CASES OF BASILAR
IMPRESSION

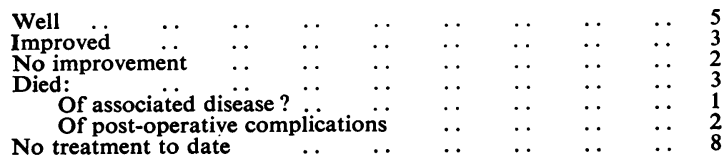

In the first place, many cases of basilar impression (more than indicated in this selected series) show no neurological complications. Of those that do, in some the complications are of minor degree and are not progressive, or may subside, either spontaneously or with rest. Some severe cases may be unsuitable for surgery. In others, concomitant lesions may be more important, though the basilar impression may contribute to the syndrome.

In a few special cases, immobilization in a collar may be worth a trial. Even where there is not abnormal mobility, as in Case 13, stress from movement or friction at the level of the foramen magnum may play as great a part in the production of the neurological syndrome as simple compression. An analogy may be drawn with cases of cervical spondylosis. The lasting benefit from temporary immobilization in Case 13 was striking. The more adequate immobilization of the occipito-atlantal joint by a Minerva type of plaster collar (as opposed to a short collar under the chin) is worth stressing.

Traction was used in one bedridden patient (Case 18). Serial radiographs showed minimal correction of the deformity, and benefit from this treatment was attributed mainly to the immobilization procured by this method in conjunction with a plaster bed. Immobilization was a most useful first stage in treatment in this neurologically advanced case, before the major procedure of operation. Such a preliminary manœuvre might be useful in other severe cases, though no reduction of bony deformity could be expected, and it would not be likely to obviate the need for surgery.

Surgical decompression of the foramen magnum region is the logical treatment in any but minor cases, where the neurological syndrome is progressive. In view of the danger of serious complications, even in cases which do not appear neurologically advanced (Cases 16, 19), this should not be left too late. The possibility of associated softtissue anomalies is a hazard (Case 15), but not necessarily a fatal one (Case 1).

The decompression, as pointed out by O'Connell and Turner (1950), must be adequate. This means laminectomy of at least the upper two cervical vertebrae and division of any underlying band of fibrous tissue (Case 4). The dura mater should be opened. In addition to providing more efficiento decompression, this allows access to any obstructing arachnoidal scars or membranes (Case 19). In viewD of the possibility of generalized compression of the posterior fossa, perhaps with upward herniation of the cerebellum and midbrain compression aso postulated by several writers, a fairly wide suboccipital decompression is desirable. This is readily obtained by a classical suboccipital decompression using a transverse incision by the superior nuchal line, but not so certain with the easier midline vertical, muscle-splitting approach. Details of operation may, of course, vary in the presence of associated lesions, such as the Arnold-Chiari malformation or subarachnoid adhesions.

Gentleness in handling these patients cannot be over-emphasized. Alarming disturbances of respiration, circulation, and state of consciousness have been encountered as immediate post-operative complications (Cases 15, 18, 19). That these were not simply post-anaesthetic complications is shown by the patient who had local anaesthesia (Case 18). The susceptibility of the medulla to movement or posture of the head in such cases was illustrated by Case 1 , who appeared at operation to have an Arnold-Chiari malformation. Although chart readings throughout the operation had shown no disturbance, respirations ceased when the patient was turned on her right side after having been put back to bed. They began again when she was put on her back. She was again carefully turned to the 
half-right position and, after another apnoea of half a minute, respiration continued spontaneously.

The short neck and increased lordosis have caused difficulty in tracheal intubation for the anaesthetic and with the airway post-operatively, the laryngeal aditus tending to fall back against the posterior pharyngeal wall.

Bronchopneumonia was a troublesome postoperative complication. This was not surprising in view of the liability to bulbar disorder, with motor and sensory loss, and reflex disturbances of the pharynx and larynx. Moreover, some of these cases had severe kyphosis and fixation of the chest with a diminished respiratory excursion. Some had weakness of the intercostal muscles and diaphragm. There appeared to be a particular susceptibility to morphine and other narcotics. This has been observed in patients with kyphosis undergoing surgical treatment for other conditions.

In addition to the post-operative complications described above, pleocytosis in the C.S.F. developed in four cases and persisted to a degree that suggested infective meningitis. In only one of these did the fluid become frankly purulent with culture of an organism, and that very late in the course of the illness, so that there remained some doubt as to whether the organisms were not secondarily introduced. Repeated lumbar puncture in any case had to be performed over a long period because of persistent reaccumulation of fluid. (This difficulty in management has been noted by O'Connell and Turner.)

A bone graft for permanent fixation (involving permanent stiffness of the neck) may sometimes be desirable. In the one patient (Case 18) in this series in whom it was used the circumstances (destruction of the atlas, presumed to be by chronic osteitis) were unusual. In most cases it is not likely to prove necessary.

\section{SUMMARY}

The lower limit of abnormality in basilar impression appears to be arbitrary, though various radiological criteria serve to identify the well-marked case.

The incidence of this condition in neurological patients is appreciable, though less than might have been expected from the previously recorded incidence in normal subjects.

Associated skeletal and soft-tissue lesions of a developmental character have been commonly found in this series of 21 cases.

Surgical treatment has been required where a neurological syndrome has been progressive-in about half the cases. Special difficulties in management have been due to disorders of the medulla, the respiratory tract, and the cerebrospinal fluid circulation.

I must acknowledge the helpful cooperation of Dr. A. M. G. Campbell, who referred for treatment many of the cases recorded here ; my thanks are also due to Dr. H. L. Hoffman, who referred others, and to Mr. G. L. Alexander, who has kindly allowed me to include in this study cases under his care.

\section{REFERENCES}

Brain, W. R. (1951). Diseases of the Nervous System, 4th ed., Oxford University Press, London

Bull, J. W. D. (1946). Proc. roy. Soc. Med., 40, 85.

Bull, J. W. D. (1946). Proc. roy. Soc. Med., 40, 85.
Chamberlain, W. E. (1939). Yale J. Biol. Med., 11, 487.

Garcin, R., and Oeconomos, D. (1953). Les Aspects Neurologiques des Malformations Congénitales de la Charnière Craniorachidienne. Masson, Paris.

Gardner, W. J., and Goodall, R. J. (1950). J. Neurosurg., 7, 199. Gustafson, W. A., and Oldberg, E. (1940). Arch. Neurol. Psychiat., Chicago, 44, 1184.

Köhler, A. (1935). Röntgenology, 2nd ed. Baillière, Tindall and Cox, London.

Lichtenstein, B. W. (1942). Arch. Neurol. Psychiat., Chicago, 47, 195. -(1943). Ibid., 49, 881 .

List, C. F. (1941). Ibid., 45, 577.

MacMahon, B., Pugh, T. F., and Ingalls, T. H. (1953). Brit. J. prev. soc. Med., 7, 211 .

Mrev. soc. Med., 7, 211. Mayo Clin., 18, 353.

O'Connell, J. E. A., and Turner, J. W. A. (1950). Brain, 73, 405.

Saunders, W. W. (1943). Radiology, 41, 589. 